\title{
Anesthesia for Robotic Thoracic Surgery
}

\author{
Javier Campos
}

Introduction

The da Vinci ${ }^{\otimes}$ Robot Surgical System ................................................................ 445

Anesthetic Implications in Robotic Thoracic Surgery ............................................. 446

Robotic-Assisted Surgery and Anesthesia for Mediastinal Masses........................... 447

Robotic-Assisted Pulmonary Lobectomy .............................................................. 448

Carbon Dioxide Insufflation During Robotic Surgery ............................................. 448

Robotic-Assisted Esophageal Surgery and Anesthetic Implications ......................... 449

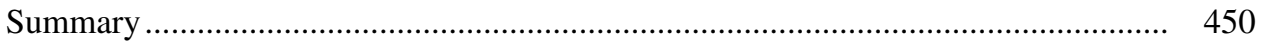

\section{Key Points}

- The management of the robotic thoracic surgical patient requires the knowledge of minimally invasive surgery techniques involving the chest.

- Familiarity with the da Vinci ${ }^{\circledR}$ robot surgical system by the anesthesiologist is mandatory.

- Management of one-lung ventilation techniques with a leftsided double-lumen endotracheal tube or an independent bronchial blocker is required, along with flexible fiberoptic bronchoscopy techniques.

- Patient positioning and prevention of complications such as nerve or crashing injuries while the robotic system is used.

- Recognition of the hemodynamic effects of carbon dioxide $\left(\mathrm{CO}_{2}\right)$ during insufflation in the chest is required.

- Potential for conversion to open thoracotomy or open procedure in the abdomen.

\section{Introduction}

Minimally invasive surgery approaches have become increasingly popular in cardiac, thoracic, and esophageal surgery [1-5]. With the introduction of robotic systems, specifically the da Vinci ${ }^{\circledR}$ robot surgical system, more than 10 years ago, a wide variety of surgical operations have been performed with some provocative results and limited defined advantages. This chapter provides an overview of the anesthetic implications and the use of the robotic system in patients undergoing mediastinal mass resection, pulmonary resections, and esophageal surgery.

\section{The da Vinci ${ }^{\circledR}$ Robot Surgical System}

The da Vinci ${ }^{\circledast}$ robot surgical system provides three dimensional (3D) video imaging plus a set of telemanipulated flexible effector instruments [6]. The system consists of three major components, a console for the operating surgeon, a patient-side cart with four interactive robotic arms, and a vision cart including optical devices for the robotic camera [7]. Figure 31.1 displays the da Vinci ${ }^{\circledR}$ robot surgical system.

In brief the surgeon operates while seated at a console and views a 3D image of the surgical fields through the vision system. The patient-side cart (the actual robot) consists of three or four robotic arms, two or three instrument arms, and one endoscope arm which houses the camera. A full range of EndoWrists (Surgical Intuitive) instruments are used to assist with the surgery. These EndoWrists provide seven degrees of motion which exceeds the capacity of a surgeon's hand in open surgery and two degrees of axial rotation to replicate human wrist-like movements. In clinical practice the first two arms, representing the surgeon's left and right hands, hold the EndoWrist instruments; a third arm positions the endoscope, the optional fourth arm, which represent the latest design in the da Vinci ${ }^{\circledR}$ robot surgical system, adds surgical capabilities by enabling the surgeon to add a third EndoWrist instrument. The surgical instruments are introduced via special ports and attached to the arms of the robot. The surgeon sitting at the console triggers highly sensitive motion sensors that transfer the surgeon's movement to the tip of the instruments. Figure 31.2 displays the console and a surgeon seated and performing robotic surgery. Figure 31.3 displays the Endo Wrist instruments during a thoracic surgical case. 


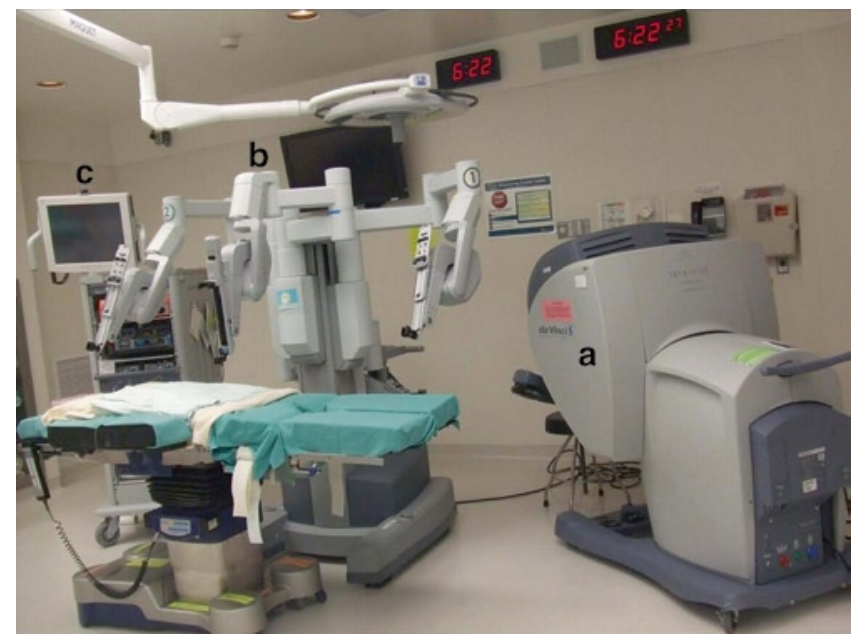

FIG. 31.1. Displays (a) the console (b) a three arm da Vinci ${ }^{\circledR}$ robot surgical system and (c) video monitor.

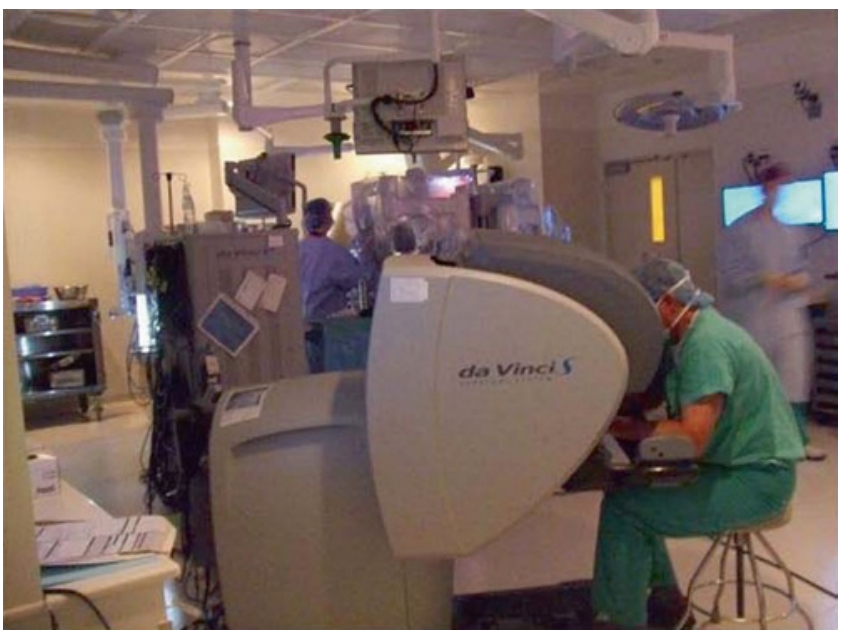

FIG. 31.2. Displays the console and a surgeon seated and performing robotic surgery.

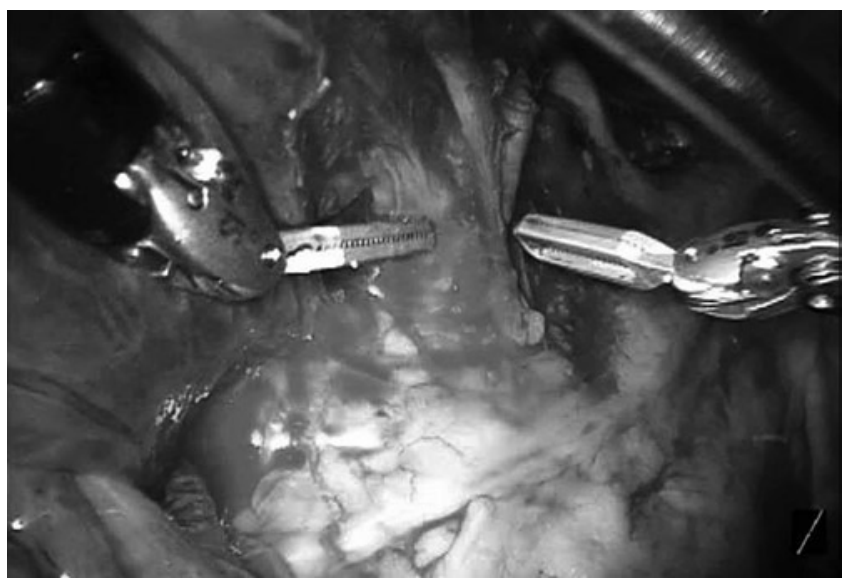

FIG. 31.3. Displays the Endo Wrist instruments during a thoracic surgical case.
TABLE 31.1. Advantages and disadvantages of robotic thoracic surgery.

Advantages of robotic thoracic surgery

- Shorter hospital length of stay

- Less pain

- Less blood loss and need for transfusion

- Minimal scarring

- Faster recovery

- Faster return to normal activities

Disadvantages

- Increasing surgical times

- Increased number of operating room personnel needed

- Potential for conversion to open procedure

- Cost and outcomes (need to be compared with other techniques)

TABLE 31.2. Surgical procedures performed in thoracic surgery with the da Vinci ${ }^{\circledR}$ robotic surgical system.

- Thymectomy

- Mediastinal mass resection

- Nissen fundoplications

- Esophageal dissections

- Esophagectomy

- Pulmonary lobectomy

Robotic surgical procedures are usually performed by two surgeons, the surgeon at the console and the table-side surgeon, who introduces the trocars and connects them with the robotic arms and changes the robotic instruments through the other ports if needed. The size of the robotic trocar is $10 \mathrm{~mm}$ for the binocular robotic camera and $8 \mathrm{~mm}$ for the instruments. Some of the potential advantages of using a robotic surgical system in thoracic surgery include: shorter hospital length of stay, less pain, less blood loss and transfusion, minimal scarring, faster recovery, and probably a faster return to normal activities [8,9]. Table 31.1 displays the advantages and disadvantages of robotic thoracic surgery. Table 31.2 displays the surgical procedures performed in thoracic surgery with the da Vinci ${ }^{\circledR}$ robot surgical system.

\section{Anesthetic Implications in Robotic Thoracic Surgery}

The basic principles applied to minimally invasive surgery of the chest (i.e., thoracoscopic surgery, see also Chap. 23) also apply to robotic-assisted thoracic surgery. The combination of patient position, management of one-lung ventilation (OLV) techniques, and surgical manipulations alter ventilation and perfusion from the dependant and nondependant or collapsed lung. The preferred method for lung isolation during robotic-assisted thoracic surgery is the use of a left-sided double-lumen endotracheal tube (DLT) because of the greater margin of safety and faster and more reliable lung collapse. Also, it provides ready access for bronchoscopic evaluation of the airway during surgical resection. 
In general, careful attention must be given to airway devices because changes in body position may cause tube migration. OLV anesthetic management is more challenging during robotic thoracic surgery due to the presence of the robot chassis that is stationed over the patient. The patient's airway is also usually located far from the anesthesia field. In some instances access to the airway, if needed, is not optimal because of the presence of the robotic arms nearby. In addition, visualization during robotic thoracic surgery may be enhanced by continuous intrathoracic carbon dioxide $\left(\mathrm{CO}_{2}\right)$ insufflation, which may increase airway pressures. When $\mathrm{CO}_{2}$ is used it should not exceed intrathoracic pressures of $10-15 \mathrm{mmHg}$. Increasing the intrathoracic pressure (i.e., $>25 \mathrm{mmHg}$ ) can compromise venous return and cardiac compliance; also the dependant lung develops higher airway pressures and ventilation can become difficult. During the surgical procedure, the $\mathrm{FiO}_{2}$ should be maintained at $100 \%$ and the peak inspiratory pressure should be kept $<30 \mathrm{~cm} \mathrm{H}_{2} \mathrm{O}$. The ventilatory parameters should be adjusted to maintain a $\mathrm{PaCO}_{2}$ at approximately $40 \mathrm{mmHg}$.

\section{Robotic-Assisted Surgery and Anesthesia for Mediastinal Masses}

Among the thoracic surgical procedures performed to date with the use of the da Vinci ${ }^{\circledR}$ robot surgical system is thymectomy [3]. Of the patients scheduled for robotic-assisted thymectomy, some have the diagnosis of myasthenia gravis because of the presence of a thymoma. Preparation of the patient for surgery includes neurological evaluation to assess the patient's neurological status and optimization of neurological conditions; continuation of anticholinesterase therapy and plasmapheresis may be indicated in some cases $[10,11]$ (see also Chap. 15). Precautions regarding anesthetic management include the proper dosing of muscle relaxants and the potential consequences of a large mediastinal mass on oxygenation and ventilation.

Positioning the patient for a thymectomy with the use of the robot system requires an optimal surgical position. In these cases, the patients are placed in an incomplete side-up position at a $30^{\circ}$ angle right or a left lateral decubitus position with the use of a beanbag. The arm of the elevated side is positioned at the patient's side as far back as possible so the surgeon can gain enough space for the robotic arms. While the robot is in use it is imperative to consider strategies to protect all pressure points and to avoid unnecessary stretching of the elevated arm because this can cause damage to the brachial plexus. Also, because the arm of the robot is in the chest cavity, a complete lung collapse must be maintained throughout the procedure. Robotic surgery with the da Vinci ${ }^{\circledR}$ robot surgical system does not allow for changes in patient position on the operating room table once the robot has been docked. Robotic thymectomy requires that the operating room table be rotated $90^{\circ}$ away from the anesthesiologist's field. For this reason access to the airway to make adjustments to the DLT during the surgery can be challenging. In some cases, a bilateral approach may be required. In these cases, the operation is performed in two stages and requires rotating the table $180^{\circ}$ to provide the surgeon access to the contralateral chest for the second stage of the operation. The anesthesiologist must be cautious during these changes to avoid problems with the airway and to ensure that the lines and monitor wires have enough slack to accommodate changes in position. The anesthesiologist must be aware during these cases about possible injury to the contralateral pleura, especially if $\mathrm{CO}_{2}$ capnothorax is being used, as the elevated intrathoracic pressure in the contralateral hemithorax can make ventilation difficult and cause cardiovascular collapse or tension pneumothorax because of malfunction of the chest tube. Special attention must be given to the patient's elevated arm and head to prevent crushing injuries with the robotic arms. A recent case report [12] showed a brachial plexus injury in an 18-year-old male after robot-assisted thoracoscopic thymectomy. In this report, the left upper limb was in slight hyperabduction. It is important to keep in mind that hyperabduction of the elevated arm to give optimal space to the operating arm of the robot can lead to a neurologic injury. Close communication between surgeon and anesthesiologist in relation to the positioning and function of the robot is mandatory, and all proper measures must be taken, including the use of soft padding and measures to avoid hyperabduction of the arm. The elevated arm should be protected by using a sling resting device. Operating room staff should always be vigilant of telescope light sources because direct contact of these devices with surgical drapes and the patient's skin can quickly cause serious burns while telescopes and cameras are being changed.

An early report by Bodner et al. [13] involving 13 patients with mediastinal masses resected with the da Vinci $^{\circledR}$ robotic surgical system showed no intraoperative complications or surgical mortality. In this series of patients, a complete thymectomy with en bloc removal of all mediastinal fat around the tumor was performed. In this report, cases were restricted to patients with a tumor size less than $10 \mathrm{~cm}$ in diameter.

In a report by Savitt et al. [4] involving 14 patients undergoing robot-assisted thymectomy, all patients received a DLT for selective lung ventilation; in addition, patients were managed with arterial and central venous pressure catheters. Complete thymectomy was performed on all 14 patients. Right-lung deflation was accomplished with selective lung ventilation and $\mathrm{CO}_{2}$ insufflation to a pressure of $10-15 \mathrm{mmHg}$ to maintain the lung away from the operative area. It is important that the anesthesiologist recognize the effects of $\mathrm{CO}_{2}$ insufflation in the thoracic cavity. The outcome of this report included no conversion to open thoracotomy, nor any intraoperative complications or deaths; the median hospital stay was 2 days with a range of 1-4 days.

In another report, Rückert et al. [9] had zero mortality and an overall postoperative morbidity rate of $2 \%$ in 106 consecutive robot-assisted thymectomies. Therefore, robotic thymectomy is a promising technique for minimally invasive surgery. 


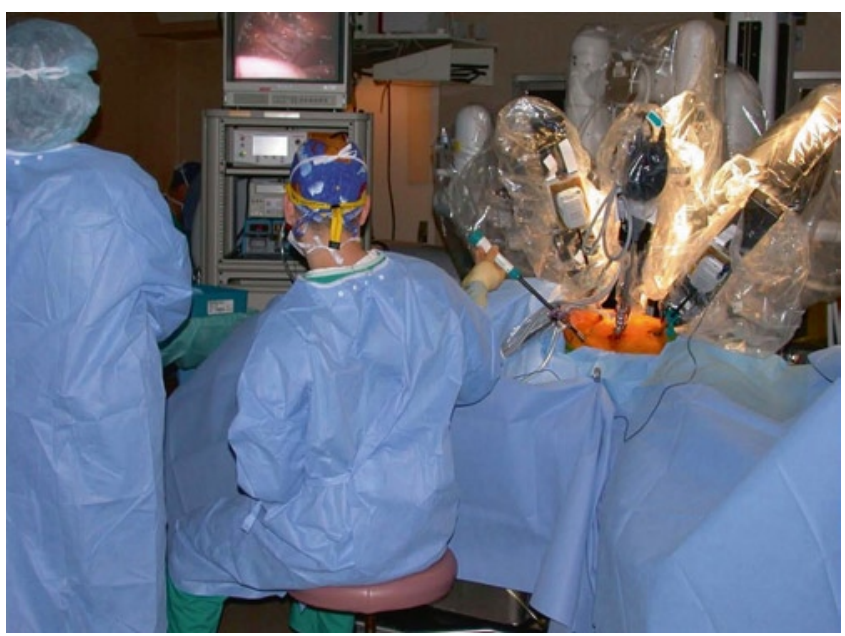

FIG. 31.4. Mediastinal mass resection.

Length of stay was shorter with robotic thymectomy when compared to the conventional approach via sternotomy. Figure 31.4 displays a case of mediastinal mass resection.

\section{Robotic-Assisted Pulmonary Lobectomy}

With the introduction of the da Vinci ${ }^{\circledR}$ robotic surgical system, there has been widespread interest in its use in minimally invasive surgery involving the chest. A report by Park et al. [14] showed robot-assisted thoracic surgical lobectomy to be feasible and safe. In the report, the operation was accomplished with the robotic system in 30 out of 34 scheduled patients. The remaining four patients required conversion to open thoracotomy. Anderson et al. [15] reported a series of 21 patients that underwent robotic lung resection for lung cancer. In this report, the 30-day mortality and conversion rate was $0 \%$. The median operating room time and blood loss was $3.6 \mathrm{~h}$ and $100 \mathrm{~mL}$. The complication rate was $27 \%$ and included atrial fibrillation and pneumonia. Gharagozloo et al. [16] reported a series of 100 consecutive robotic-assisted lobectomies for lung cancer and concluded that robotic surgery is feasible for mediastinal, hilar, and pulmonary vascular dissection during video-assisted thoracoscopy lobectomy.

Positioning the patient for a robotic lobectomy includes placing the patient over a bean bag in a maximally flexed lateral decubitus position with the elevated arm slightly extended so that the thoracic cavity can be accessed and no damage to the arm occurs during manipulation of the robotic arms. Patients undergoing robotic lobectomy must have a lung isolation device to achieve OLV. In the vast majority of these cases, a left-sided DLT is used and optimal position is achieved with the flexible fiberoptic bronchoscope [17]. In a few cases in which the airway is deemed to be difficult, an independent bronchial blocker could be used and optimal position achieved with the use of a fiberoptic bronchoscope [18]. Initial thoracic exploration is performed with conventional thoracoscopy to verify tumor location. During robot-assisted lobectomy, it is mandatory that lung collapse is achieved effectively to allow the surgeon the best field of vision and to avoid unnecessary damage to vessels or lung parenchyma.

All patients undergoing robot-assisted thoracic lobectomy should have an arterial line. The anesthesiologist must be ready for potential conversion to an open thoracotomy. In the Park report [14], three out of four cases that needed to be converted had minor bleeding; in addition, in one case lung isolation was lost, requiring an open thoracotomy. It is mandatory that the anesthesiologist involved in these cases have experience in placing a DLT [19] and can guarantee optimal position with the aid of flexible fiberoptic bronchoscope. Using intraoperative fiberoptic bronchoscopy to make adjustments to the DLT during surgery is challenging because the table is rotated $180^{\circ}$ away from the anesthesiologist's field. The chassis of the robot is often positioned over the patients head leaving a very small area for the anesthesiologist to access the airway.

A recent report by Gharagozloo et al. [16] involving 100 patients who underwent lobectomy and complete mediastinal nodal dissection for early stage lung cancer (stage I and II) with the robotic system reported one nonemergent conversion to open thoracotomy. In this report, postoperative analgesia was managed with the infusion of a local anesthetic ( $0.5 \%$ bupivacaine, $4 \mathrm{~mL} / \mathrm{h})$ through catheters placed in a subpleural tunnel encompassing intercostal spaces 2 through 8. All patients were extubated in the operating room. Mean operating room time was $216 \mathrm{~min}$ (range 173-369). Overall mortality within 30 days was $4.9 \%$, and median length of stay was 4 days. Postoperative complications included atrial fibrillation in four cases, prolonged air leak in two cases, and pleural effusion requiring drainage in two cases - complications that are not different from those occurring with video thoracoscopic surgery. Although lobectomy can be performed via robot-assisted surgery, the advantages at present are not well defined. In contrast, the increasing surgical times, the increased number of operating room personnel needed, and the cost and outcomes of robotic surgery need to be studied and compared with thoracoscopic lobectomy.

\section{Carbon Dioxide Insufflation During Robotic Surgery}

Continuous low-flow insufflation of $\mathrm{CO}_{2}$ has been demonstrated as an aid for surgical exposure during minimally invasive thoracic procedures. It has been used as the only means of providing surgical exposure to the thoracic cavity (during two-lung ventilation for VATS), or more frequently in conjunction with a DLT or an independent bronchial blocker and OLV. The compression of the lung parenchyma by $\mathrm{CO}_{2}$ acts as a retractor [20].

A study by Ohtsuka et al. [21] involving 38 patients undergoing minimally invasive internal mammary harvest during cardiac surgery found significant increases in mean central 
venous pressure, pulmonary artery pressure, and the pulmonary artery wedge pressure. They also found that with insufflation of the right hemithorax, but not the left side, slight decreases were noted in the mean arterial blood pressure and cardiac index. They concluded that the hemodynamic effect from continuous insufflation of $\mathrm{CO}_{2}$ at $8-10 \mathrm{mmHg}$ for $30-40 \mathrm{~min}$ is mild in both hemithoraces, although the impact is greater on the right. This information was supported by another study [22]. This study involving 20 patients undergoing thoracoscopic sympathectomy and concluded that compared to the left side hemithorax the impact of $\mathrm{CO}_{2}$ insufflation on the vena cava and the right atrium during right-sided procedures was associated with reduction of venous return and low cardiac index and stroke volume. The impact of $\mathrm{CO}_{2}$ insufflation on the respiratory system has also been studied. El-Dawlatly et al. [23] reported a significant pressure-dependent increase in peak airway pressure and a decrease in dynamic lung compliance but no difference in tidal volume or minute ventilation during volume-controlled ventilation.

Insufflation of $\mathrm{CO}_{2}$ should only be started after initial thoracoscopic evaluation has ruled out that the port of insufflation has not compromised a vascular structure or the lung parenchyma. Communication between the surgeon, anesthesiologist, and operating room personnel is crucial at this point. Insufflation is ideally started at low pressures of $4-5 \mathrm{mmHg}$ and is gradually increased while monitoring the patient's vital signs. The anesthesiologist should always be aware of the possibility of gas embolization during these cases. In the case of sudden cardiac collapse, the $\mathrm{CO}_{2}$ flow should be discontinued immediately. Ventilation during $\mathrm{CO}_{2}$ insufflation should be titrated to keep adequate oxygenation and a normal $\mathrm{PaCO}_{2}$ and $\mathrm{pH}$. Also, damage to the contralateral pleura may occur resulting in $\mathrm{CO}_{2}$ flow to the contralateral chest, making ventilation difficult and also causing hemodynamic compromise, along with the potential development of subcutaneous emphysema.

\section{Robotic-Assisted Esophageal Surgery and Anesthetic Implications}

Transthoracic esophagectomy with extended lymph node dissection is associated with higher morbidity rates than transhiatal esophagectomy. Esophagectomy is a palliative and potentially curative treatment for esophageal cancer. Minimally invasive esophagectomy has been performed to lessen the biological impact of surgery and potentially reduce pain. The initial esophagectomy experience with the da $\mathrm{Vinci}^{\circledR}$ robot surgical system involved a patient who had a thoracic esophagectomy with wide celiac axis lymphadenectomy. The case was reported by Kernstine et al. [24] and had promising results. Thereafter another report using the using the da Vinci ${ }^{\circledR}$ robot surgical system has been published of 6 patients undergoing esophagectomy without intraoperative complications [25]. The surgical approach in this report was performed from the right side of the chest. A left-sided DLT was used to selectively collapse the right lung while, at the same time, ventilation was maintained in the left lung.

In a report by Hillegersberg et al. [26] involving 21 consecutive patients with esophageal cancer who underwent robotassisted thoracoscopic esophagolymphadenectomy, 18 were completed thoracoscopically and 3 required open procedures (because adhesions or intraoperative hemorrhage). In this case series report, all patients received a left-sided DLT and a thoracic epidural catheter as part of their anesthetic management. Positioning of these patients was in a left lateral decubitus position, and the patient was tilted $45^{\circ}$ towards the prone position. Once the robotic thoracoscopic phase was completed, the patient was then put in supine position and a midline laparotomy was performed. A cervical esophagogastrostomy was performed in the neck for the completion of surgery.

Of interest in this series is the fact that pulmonary complications occurred in the first 10 cases $(60 \%)$, caused primarily by left-sided pneumonia and associated acute respiratory distress syndrome in 3 patients $(33 \%)$. These complications were probably related to barotrauma to the left lung (ventilated lung) attributed to high tidal volumes and high peak inspiratory pressures. In the 11 patients that followed, the same authors modified their ventilatory setting to administer continuous positive airway pressure ventilation $5 \mathrm{~cm} \mathrm{H}_{2} \mathrm{O}$ during single-lung ventilation and pressure-controlled ventilation was used; with this approach the respiratory complication rate was reduced to $32 \%$.

A recent report by Kim et al. [27] described 21 patients who underwent robotic-assisted thoracoscopic esophagectomy performed in a prone position with the use of a Univent ${ }^{\circledR}$ bronchial blocker tube (Fuji Systems Corp, Tokyo Japan). All thoracoscopic procedures were completed with robotic-assisted techniques followed by a cervical esophagogastrostomy. In Kim's report, major complications included anastomotic leakage in 4 patients, vocal cord paralysis in 6 patients, and intraabdominal bleeding in 1 patient. The prone position led to an increase in central venous pressure and mean pulmonary arterial pressure and a decrease in static lung compliance. The overall conclusion from this report is that robotic assistance esophagectomy in the prone position is technically feasible and safe. Others have reported a robotic-assisted transhiatal esophagectomy technique feasible and safe as well [28].

Another study [5] involved 14 patients who underwent esophagectomy using the da Vinci ${ }^{\circledR}$ robot surgical system in different surgical stages. It showed that for a complete robotic esophagectomy including laparoscopic gastric conduct, the operating room time was an average of $11 \mathrm{~h}$ with a console time by the surgeon of $5 \mathrm{~h}$, and an estimated mean blood loss of $400 \pm 300 \mathrm{~mL}$. In this report after the robotic thoracoscopic part of the surgery was accomplished with the patient in the lateral decubitus position, patients were then placed in supine position and reintubated, and the DLT was replaced with a single-lumen endotracheal tube. The head of each patient was turned upward and to the right, exposing the left neck for the cervical part of the operation. Among the pulmonary 


\begin{tabular}{|c|c|c|c|c|}
\hline References & $n=$ cases & Operation & Intraoperative complications & Postoperative complications \\
\hline Rea et al. [3] & 33 & Thymectomy & 0 & $\begin{array}{l}\text { Chylothorax } n=1 \\
\text { Hemothorax } n=1\end{array}$ \\
\hline Savitt et al. [4] & 15 & Mediastinal mass resection & 0 & Atrial fibrillation $n=1$ \\
\hline Kernstine et al. [5] & 14 & Esophagectomy & Conversion to open procedure $n=1$ & $\begin{array}{l}\text { Thoracic duct leak } n=3 \\
\text { Vocal cord paralysis } n=3 \\
\text { Atrial fibrillation } n=5\end{array}$ \\
\hline Rückert et al. [9] & 106 & Thymectomy & Bleeding $n=1$ & Phrenic nerve injury $n=1$ \\
\hline Pandey et al. [12] & 1 & Thymectomy & - & Brachial plexus injury \\
\hline Bodner et al. [13] & 14 & Mediastinal mass resection & $\mathrm{cos}^{\circ} \mathrm{s}$ & $\begin{array}{l}\text { Postoperative hoarseness due to lesion } \\
\text { to left laryngeal recurrent nerve }\end{array}$ \\
\hline Park et al. [14] & 34 & Lobectomy & $\begin{array}{l}\text { Conversion to open thoracotomy } n=3 \\
\text { Lack lung isolation } n=1\end{array}$ & $\begin{array}{l}\text { Supraventricular arrhythmia } n=6 \\
\text { Bleeding } n=1 \\
\text { Air leak } n=1\end{array}$ \\
\hline Gharagozloo et al. [16] & 100 & Lobectomy & 0 & $\begin{array}{l}\text { Atrial fibrillation } n=4 \\
\text { Air leak } n=2 \\
\text { Bleeding } n=1 \\
\text { Pleural effusion } n=2\end{array}$ \\
\hline Van Hillegersberg et al. [26] & 21 & Esophagectomy & Conversion to open procedure $n=3$ & $\begin{array}{l}\text { Pulmonary complication } 60 \% \\
\text { first } 10 \text { cases } \\
\text { Pulmonary complication } 32 \% \text {, } \\
11 \text { patients }\end{array}$ \\
\hline Kim et al. [27] & 21 & Esophagectomy & Bleeding $n=1$ & $\begin{array}{l}\text { Anastomotic leakage } n=4 \\
\text { Vocal cord paralysis } n=6\end{array}$ \\
\hline
\end{tabular}

complications in the postoperative period, arterial fibrillation occurred in 5 out of 14 patients.

In Kernstine's report [5] among the recommendations to improve efficiency in these cases is the "use of an experienced anesthesiologist who can efficiently intubate and manage single-lung ventilation and hemodynamically support the patient during the procedure." This follows what Nifong and Chitwood [19] have reported in their editorial views regarding anesthesia and robotics: that a team approach with expertise in these procedures involving nurses, anesthesiologists, and surgeons with an interest in robotic procedures is required.

The data on robotic-assisted esophagectomy suggest that the procedure is safe, feasible, and associated with preoperative outcomes similar to open and minimally invasive esophagectomy. No data, however, demonstrate improved outcomes in terms of operative morbidity, pain, operative time, or total costs [29]. Table 31.3 displays the complications of roboticassisted thoracic surgery involving the mediastinum lung and esophagus.

\section{Summary}

The use of the da Vinci ${ }^{\circledR}$ robot surgical system in thoracic and esophageal surgery continues to gain acceptance. Although its use has reduced surgical scarring and decreased length of stay, specific indications for use in these areas need to be determined. All reports to date describe the use of lung isolation devices, most often a left-sided DLT, as part of the intraoperative management of thoracic surgery patients to facilitate surgical exposure. In addition, because the surgical approach varies depending on the thoracic procedure, optimal positioning is not standard, and varies among the specific surgical procedures. Vigilance is required with patients' elevated arms to avoid nerve injuries or crush injuries from the robotic arms. Continuous low-flow insufflation of $\mathrm{CO}_{2}$ has been used as an aid for surgical exposure during minimally invasive thoracic procedures. The potential to convert to an open thoracotomy requires preparation by the surgical team and anesthesiologist. The use of the da Vinci ${ }^{\circledR}$ robot surgical system is expected to grow in the years to come $[30,31]$. Prospective studies are needed to define the specific advantages of this robotic system.

\section{References}

1. Tatooles AJ, Pappas PS, Gordon PJ, Slaughter MS. Minimally invasive mitral valve repair using the da Vinci robotic system. Ann Thorac Surg. 2004;77:1978-82.

2. Nifong LW, Chitwood WR, Pappas PS, Smith CR, Argenziano M, Starnes VA, et al. Robotic mitral valve surgery: a United States multicenter trial. J Thorac Cardiovasc Surg. 2005;129:1395-404.

3. Rea F, Marulli G, Bortolotti L, Feltracco P, Zuin A, Sartori F. Experience with the "da Vinci" robotic system for thymectomy in patients with myasthenia gravis. Ann Thorac Surg. 2006;8:455-9.

4. Savitt MA, Gao G, Furnary AP, Swanson J, Gately HL, Handy JR. Application of robotic-assisted techniques to the surgical evaluation and treatment of the anterior mediastinum. Ann Thorac Surg. 2005;79:450-5.

5. Kernstine KH, DeArmond DT, Shamoun DM, Campos JH. The first series of completely robotic esophagectomies with three-field lymphadenectomy: initial experience. Surg Endosc. 2007;21:2285-92.

6. Mack MJ. Minimally invasive and robotic surgery. JAMA. 2001;285:568-72. 
7. Campos JH. An update on robotic thoracic surgery and anesthesia. Curr Opin Anaesthesiol. 2010;23:1-6.

8. Bodner J, Wykypiel H, Wetscher G, Schmid T. First experiences with the da Vinci operating robot in thoracic surgery. Eur J Cardiothorac Surg. 2004;25:844-51.

9. Rückert JC, Ismail M, Swierzy M, Sobel H, Rogalla P, Meisel A, et al. Thoracoscopic thymectomy with the da Vinci robotic system for myasthenia gravis. Ann NY Acad Sci. 2008;1132:329-35.

10. Baraka A. Onset of neuromuscular block in myasthenic patients. Br J Anaesth. 1992;69:227-8.

11. Abel M, Eisenkraft JB. Anesthetic implications of myasthenia gravis. Mt Sinai J Med. 2002;69:31-7.

12. Pandey R, Elakkumanan LB, Garg R, Jyoti B, Mukund C, Chandralekha, et al. Brachial plexus injury after robotic-assisted thoracoscopic thymectomy. J Cardiothorac Vasc Anesth 2009;23:584-6.

13. Bodner J, Wykypiel H, Greiner A, Kirchmayr W, Freund MC, Margreiter R, et al. Early experience with robot-assisted surgery for mediastinal masses. Ann Thorac Surg. 2004;78:259-65.

14. Park BJ, Flores RM, Rusch VW. Robotic assistance for videoassisted thoracic surgical lobectomy: technique and initial results. J Thorac Cardiovasc Surg. 2006;131:54-9.

15. Anderson CA, Filsoufi F, Aklog L, Farivar RS, Byrne JG, Adams DH. Robotic-assisted lung resection for malignant disease. Innovations. 2007;2:254-8.

16. Gharagozloo F, Margolis M, Tempesta B, Strother E, Najam F. Robot-assisted lobectomy for early-stage lung cancer: report of 100 consecutive cases. Ann Thorac Surg. 2009;88:380-4.

17. Campos JH. Update on tracheobronchial anatomy and flexible fiberoptic bronchoscopy in thoracic anesthesia. Curr Opin Anaesthesiol. 2009;22:4-10.

18. Campos JH. Progress in lung separation. Thorac Surg Clin. 2005;15:71-83.

19. Nifong LW, Chitwood Jr WR. Challenges for the anesthesiologist: robotics? Anesth Analg. 2003;96:1-2.

20. Wolfer RS, Krasna MJ, Hasnain JU, McLaughlin JS. Hemodynamic effects of carbon dioxide insufflation during thoracoscopy. Ann Thorac Surg. 1994;58:404-7.
21. Ohtsuka T, Nakajima J, Kotsuka Y, Takamoto S. Hemodynamic response to intrapleural insufflation with hemipulmonary collapse. Surg Endosc. 2001;15:1327-30.

22. El-Dawlatly AA, Al-Dohayan A, Samarkandi A, Algahdam F, Atef A. Right vs left side thoracoscopic sympathectomy: effects of carbon dioxide insufflation on haemodynamics. Ann Chir Gynaecol. 2001;90:206-8.

23. El-Dawlatly AA, Al-Dohayan A, Abdel-Meguid ME, Turkistani A, Alotaiby WM, Abdelaziz EM. Variations in dynamic lung compliance during endoscopic thoracic sympathectomy with carbon dioxide insufflation. Clin Auton Res. 2003;13 Suppl 1:I94-7.

24. Kernstine KH, DeArmond DT, Karimi M, Van Natta TL, et al. The robotic, 2-stage, 3-field esophagolymphadenectomy. J Thorac Cardiovasc Surg. 2004;127:1847-9.

25. Bodner JC, Zitt M, Ott H, Wetscher GJ, et al. Robotic-assisted thoracoscopic surgery (RATS) for benign and malignant esophageal tumors. Ann Thorac Surg. 2005;80:1202-6.

26. van Hillegersberg R, Boone J, Draaisma WA, Broeders IA, et al. First experience with robot-assisted thoracoscopic esophagolymphadenectomy for esophageal cancer. Surg Endosc. 2006;20:1435-9.

27. Kim DJ, Hyung WJ, Lee CY, Lee JG, Haam SJ, Park IK, et al. Thoracoscopic esophagectomy for esophageal cancer: feasibility and safety of robotic assistance in the prone position. $\mathrm{J}$ Thorac Cardiovasc Surg. 2010;139:53-9.

28. Gutt CN, Bintintan VV, Köninger J, Müller-Stich BP, Reiter M, Büchler MW. Robotic-assisted transhiatal esophagectomy. Langenbecks Arch Surg. 2006;391:428-34.

29. Watson TJ. Robotic esophagectomy: is it an advance and what is the future? Ann Thorac Surg. 2008;85:757-9.

30. Czibik G, D’Ancona G, Donias HW, Karamanoukian HL. Robotic cardiac surgery: present and future applications. J Cardiothorac Vasc Anesth. 2002;16:495-501.

31. Hubens G, Ruppert M, Balliu L, Vaneerdeweg W. What have we learnt after two years working with the da Vinci robot system in digestive surgery? Acta Chir Belg. 2004;104:609-14. 